\title{
DEVELOPMENT OF SINGULARITIES IN SOLUTIONS OF A HYPERBOLIC SYSTEM
}

\author{
S. A. MESSAOUDI \\ (Received 10 November 2000 and in revised form 20 February 2001)
}

\begin{abstract}
We consider a special type of a hyperbolic system and show that classical solutions blow up in finite time even for small initial data.
\end{abstract}

2000 Mathematics Subject Classification. 35L45.

1. Introduction. For the system of nonlinear elasticity

$$
u_{t}(x, t)=\varphi(v(x, t)) v_{x}(x, t), \quad v_{t}(x, t)=u_{x}(x, t),
$$

it is well known that $C^{1}$-solutions break down in finite time however smooth and small the initial data are. This was shown by Lax [4] in 1964. In his work, the author studied (1.1), for $\varphi>0$ and $\varphi^{\prime}>0$, and established a blowup result. MacCamy and Mizel [7] in 1967 considered the same system and proved a similar result, allowing $\varphi^{\prime}$ to change sign. They also showed, under appropriate conditions on $\varphi$, that there are $x$-intervals, for which the solution must exist for all time even though it blows up for values of $x$ outside these intervals.

Messaoudi [9] discussed the following system:

$$
u_{t}(x, t)=\alpha(x) \varphi(v(x, t)) v_{x}(x, t), \quad v_{t}(x, t)=u_{x}(x, t)
$$

which models a transverse motion of a string with variable density. He showed that $C^{1}$-solutions develop singularities in finite time if the initial data are taken with large enough gradients. He also discussed, in [8], a system with dissipation of the form

$$
\theta_{t}+c(\theta) q_{x}=0, \quad q_{t}+\sigma(\theta) \theta_{x}=-\lambda(\theta) q,
$$

which describes heat propagation in materials that predict finite propagation speed. This phenomenon is called second sound. Here $\theta$ is the difference temperature and $q$ is the heat flux. He studied the Cauchy problem and proved a blowup result of the classical solutions. We should note that, for $\lambda$ constant and $c(\theta)=-1,(1.3)$ reduces to a system describing steady shearing flows in nonlinear viscoelastic fluids. This problem was studied by Slemrod [11] and a blowup result for classical solutions has been established. A similar problem was also discussed by Nishibata [10], Kosiński [3], and Zheng [12] and results concerning global existence and nonexistence have been accomplished. 
For more general systems, it is worth mentioning the work of Li et al. [6], in which they discussed

$$
u_{t}(x, t)=A(u(x, t)) u_{x}(x, t),
$$

associated with decaying initial data. Here $u: I \times(0, T) \rightarrow \mathbb{R}^{n}$ is a vector-valued function, $A$ is an $(n \times n)$-matrix, and $I$ is an interval (bounded or unbounded). They proved a global $C^{1}$-solution for the Cauchy problem if, in addition to the local strict hyperbolicity condition, (1.4) is weakly linearly degenerate and the initial data satisfy, for $\mu>0$, $\sup _{x}\left\{(1+|x|)^{1+\mu}\left|u_{0}^{\prime}(x)\right|+\left|u_{0}(x)\right|\right\}$ is small enough. They also established a blowup result to $C^{1}$-solutions for nonweakly linearly degenerate systems. As they pointed out, their work generalizes their result of [5] to the case of initial data with no compact support but they possess certain decay properties.

In this work, we are concerned with a quasilinear hyperbolic system of the form

$$
u_{t}(x, t)=\varphi\left(\frac{v(x, t)}{1+a u(x, t)}\right) v_{x}(x, t), \quad v_{t}(x, t)=u_{x}(x, t)
$$

where the constant $a \neq 0$. In addition to its importance from the mathematical technique point of view, this system can be regarded as a relative generalization of the one-dimensional wave equation in the sense if $a=0,(1.5)$ reduces to (1.1). We will consider (1.5) together with initial conditions and show that $C^{1}$-solutions blowup even for small initial data. Our result cannot be directly deduced from the results of [6] since we do not impose the same conditions regarding the size and the regularity of the initial data (cf. [6, Theorem 1.2] and Theorem 3.1 below). This work is divided into two parts. In part one we state, without proof, a local existence theorem. In part two our main result is stated and proved.

2. Local existence. We consider the following Cauchy problem

$$
\begin{gathered}
u_{t}(x, t)=\varphi\left(\frac{v(x, t)}{1+a u(x, t)}\right) v_{x}(x, t), \\
v_{t}(x, t)=u_{x}(x, t), \quad \forall x \in \mathbb{R}, t>0, \\
u(x, 0)=u_{0}(x), \quad v(x, 0)=v_{0}(x), \quad \forall x \in \mathbb{R},
\end{gathered}
$$

where $a \neq 0$ and $\varphi$ is a function satisfying

$$
\varphi(\xi) \geq \beta>0, \quad \forall \xi \in \mathbb{R} .
$$

Proposition 2.1. Assume that $\varphi$ is a $C^{1}$ function satisfying (2.4) and let $u_{0}$ and $v_{0}$ in $H^{2}(\mathbb{R})$ be given such that

$$
\left|1+\operatorname{au}_{0}(x)\right| \geq \lambda>0, \quad \forall x \in \mathbb{R} .
$$

Then the problem (2.1), (2.2), and (2.3) has a unique local solution ( $u, v)$, on a maximal time interval $[0, T)$, satisfying

$$
u, v \in C\left([0, T), H^{2}(\mathbb{R})\right) \cap C^{1}\left([0, T), H^{1}(\mathbb{R})\right) .
$$


This result can be proved by applying a classical energy argument [1] or the nonlinear semigroup theory [2].

REMARK 2.2. The functions $u, v$ are $C^{1}$ functions by the standard Sobolev embedding theory.

3. Formation of singularities. We introduce the quantities and the differential operators

$$
\begin{aligned}
r & :=\frac{1}{a} \ln |1+a u|+\int_{0}^{v /(1+a u)} \alpha(\xi) d \xi \\
s & :=\frac{1}{a} \ln |1+a u|-\int_{0}^{v /(1+a u)} \beta(\xi) d \xi \\
\partial_{t} & :=\frac{\partial}{\partial t}-\rho\left(\frac{v}{1+a u}\right) \frac{\partial}{\partial x}, \\
D_{t} & :=\frac{\partial}{\partial t}+\rho\left(\frac{v}{1+a u}\right) \frac{\partial}{\partial x},
\end{aligned}
$$

where

$$
\rho(\xi)=\sqrt{\varphi(\xi)}, \quad \alpha(\xi)=\frac{\sqrt{\varphi(\xi)}}{1+a \xi \sqrt{\varphi(\xi)}}, \quad \beta(\xi)=\frac{\sqrt{\varphi(\xi)}}{1-a \xi \sqrt{\varphi(\xi)}}
$$

The following lemma shows, for initial data appropriately chosen, that $r$, s, and $\rho$ are well defined and $|v(x, t) /(1+a u(x, t))|$ is uniformly bounded.

THEOREM 3.1. Let $a$ and $\varphi$ be as in Proposition 2.1. Then there exist initial data in $H^{2}(\mathbb{R})$ satisfying (2.5), for which

$$
\left|\frac{a v(x, t)}{1+a u(x, t)} \sqrt{\varphi\left(\frac{v(x, t)}{1+a u(x, t)}\right)}\right|<1, \quad|1+a u(x, t)|>0,
$$

and $|v(x, t) /(1+a u(x, t))|$ is uniformly bounded on $\mathbb{R} \times[0, T)$.

Proof. We first choose $\delta>0$ such that if

$$
\left|u_{0}(x)\right|<\delta, \quad\left|v_{0}(x)\right|<\delta, \quad \forall x \in \mathbb{R}
$$

then

$$
\left|\frac{a v_{0}(x)}{1+a u_{0}(x)} \sqrt{\varphi\left(\frac{v_{0}(x)}{1+a u_{0}(x)}\right)}\right|<1, \quad\left|1+a u_{0}(x)\right|>0, \forall x \in \mathbb{R}
$$

Of course, this is possible by taking $\delta$ small enough. Then the continuity of $u, v$, and $\varphi$ implies that there exists $T^{\prime} \leq T$, such that (3.3) holds on $\mathbb{R} \times\left[0, T^{\prime}\right)$. Let $T_{0}:=\sup \left\{T^{\prime}\right.$ : (3.3) holds for all $\left.x \in \mathbb{R}, t \in\left[0, T^{\prime}\right)\right\}$. We have two cases, either $T_{0}=T$, this completes 
the proof. Or $T_{0}<T$; in this case we estimate

$$
\begin{aligned}
\partial_{t} r= & \frac{u_{t}}{1+a u}+\alpha\left[\frac{v_{t}}{1+a u}-\frac{v}{(1+a u)^{2}} a u_{t}\right] \\
& -\rho\left[\frac{u_{x}}{1+a u}+\alpha \frac{v_{x}}{1+a u}-\alpha \frac{v}{(1+a u)^{2}} a u_{x}\right] \\
= & \frac{1}{1+a u}\left[\left(1-a \alpha \frac{v}{1+a u}\right) u_{t}-\alpha \rho v_{x}\right] \\
& +\frac{1}{1+a u}\left[\alpha v_{t}-\rho\left(1-a \alpha \frac{v}{1+a u}\right) u_{x}\right], \quad \forall x \in \mathbb{R}, t \in\left[0, T_{0}\right) .
\end{aligned}
$$

We recall that, unless otherwise stated, $\alpha, \beta, \rho$, and $\varphi$ are functions of $v /(1+a u)$. By noting that $\alpha \rho=(1-a \alpha v /(1+a u)) \varphi,(1-a \alpha 1-a \alpha v /(1+a u)) \rho=\alpha$, and using (2.1) and (2.2), we obtain

$$
\partial_{t} r=0, \quad \forall x \in \mathbb{R}, t \in\left[0, T_{0}\right)
$$

Similar calculations also yield

$$
D_{t} s=0, \quad \forall x \in \mathbb{R}, t \in\left[0, T_{0}\right) .
$$

Therefore, on $\mathbb{R} \times\left[0, T_{0}\right), r$ and $s$ remain constant along backward and forward characteristics, respectively; hence $\|r\|_{\infty}=\left\|r_{0}\right\|_{\infty}$ and $\|s\|_{\infty}=\left\|s_{0}\right\|_{\infty}$. It is easy to see that

$$
r(x, t)-s(x, t)=\phi\left(\frac{v(x, t)}{1+a u(x, t)}\right), \quad \forall x \in \mathbb{R}, t \in\left[0, T_{0}\right),
$$

where $\phi(\tau)=2 \int_{0}^{\tau} \sqrt{\varphi(\xi)} /\left(1-a^{2} \xi^{2} \varphi(\xi)\right) d \xi$ is strictly monotone and continuous at least in a neighborhood of zero, so it admits a continuous inverse $\psi$ near zero. Since the function $g(\xi)=1-a^{2} \xi^{2} \varphi(\xi)$ is continuous and $g(0)=1$, one can choose $\gamma$ so that $g(\xi) \geq \varepsilon>0$, for all $|\xi|<\gamma$ and choose $\delta_{1}>0$ so that $|\psi(\tau)|<\gamma$, for all $|\tau|<\delta_{1}$. Therefore, by choosing $\delta$ small enough so that (3.4) holds and $\left\|r_{0}\right\|_{\infty}+\left\|s_{0}\right\|_{\infty}<\delta_{1}$, we get

$$
|r(x, t)-s(x, t)| \leq\left\|r_{0}\right\|_{\infty}+\left\|s_{0}\right\|_{\infty}<\delta_{1}
$$

consequently

$$
\left|\frac{v(x, t)}{1+a u(x, t)}\right|=|\psi(r-s)|<\gamma
$$

which yields

$$
\left|\frac{a v(x, t)}{1+a u(x, t)} \sqrt{\varphi\left(\frac{v(x, t)}{1+a u(x, t)}\right)}\right| \leq 1-\varepsilon<1, \quad \forall x \in \mathbb{R}, t \in\left[0, T_{0}\right] .
$$

We then use (3.1), the boundedness of $r$, and the fact that $1+a \xi \sqrt{\varphi(\xi)} \geq \varepsilon$ to conclude that $\ln |1+a u|$ is bounded on $\mathbb{R} \times\left[0, T_{0}\right]$; hence $|1+a u|>0$. Again by continuity, there 
exists $T_{1}>T_{0}$ such that (3.3) holds on $\mathbb{R} \times\left[0, T_{1}\right)$. This contradicts the maximality of $T_{0}$; hence $T_{0}$ must be equal to $T$. Therefore (3.3) and (3.11) hold. This completes the proof.

TheOrem 3.2. Assume that, in addition to (2.4), $\varphi$ satisfies $\varphi^{\prime}(0)>0$. Then there exist initial data $u_{0}, v_{0}$ in $H^{2}(\mathbb{R})$ satisfying (3.4), for which the solution of the problem (2.1), (2.2), and (2.3) blows up in finite time.

Proof. We take an $x$-partial derivative of (3.7) to get

$$
\left(\partial_{t} r\right)_{x}=r_{x t}-\rho r_{x x}-r_{x} \rho_{x}=0
$$

which, in turn, implies

$$
\partial_{t} r_{x}=r_{x} \rho_{x}=\frac{\varphi^{\prime}}{2 \sqrt{\varphi}} r_{x} \frac{\partial}{\partial x}\left(\frac{v}{1+a u}\right)
$$

We then use

$$
r_{x}=\frac{u_{x}}{1+a u}+\alpha \cdot \frac{\partial}{\partial x}\left(\frac{v}{1+a u}\right), \quad s_{x}=\frac{u_{x}}{1+a u}-\beta \cdot \frac{\partial}{\partial x}\left(\frac{v}{1+a u}\right),
$$

and substitute in (3.14) to arrive at

$$
\begin{aligned}
\partial_{t} r_{x} & =\frac{\varphi^{\prime}}{2 \sqrt{\varphi}(\alpha+\beta)} r_{x}\left(r_{x}-s_{x}\right) \\
& =\frac{\varphi^{\prime}}{4 \varphi}\left(1-a^{2}\left(\frac{v}{1+a u}\right)^{2} \varphi\right) r_{x}^{2}-\frac{\varphi^{\prime}}{4 \varphi}\left(1-a^{2}\left(\frac{v}{1+a u}\right)^{2} \varphi\right) r_{x} s_{x}
\end{aligned}
$$

To handle the last term in (3.16), we set $W:=\varphi^{1 / 4} r_{x}$ and substitute in (3.16), to get

$$
\begin{aligned}
\partial_{t} W= & \varphi^{1 / 4} \frac{\varphi^{\prime}}{4 a \varphi}\left(1-a^{2}\left(\frac{v}{1+a u}\right)^{2} \varphi\right) r_{x}^{2}-\varphi^{1 / 4} \frac{\varphi^{\prime}}{4 a \varphi}\left(1-a^{2}\left(\frac{v}{1+a u}\right)^{2} \varphi\right) r_{x} s_{x} \\
& +\frac{1}{4} \varphi^{-3 / 4} \varphi^{\prime} r_{x} \partial_{t}\left(\frac{v}{1+a u}\right) .
\end{aligned}
$$

By using (2.1) and (2.2), we see that

$$
\begin{aligned}
\partial_{t}\left(\frac{v}{1+a u}\right) & =\frac{(1+a u)\left(v_{t}-\sqrt{\varphi} v_{x}\right)-a v\left(u_{t}-\sqrt{\varphi} u_{x}\right)}{(1+a u)^{2}} \\
& =\frac{(1+a u)\left(u_{x}-\sqrt{\varphi} v_{x}\right)-a v\left(\varphi v_{x}-\sqrt{\varphi} u_{x}\right)}{(1+a u)^{2}} \\
& =\frac{\left(u_{x}-\sqrt{\varphi} v_{x}\right)(1+a u+a \sqrt{\varphi} v)}{(1+a u)^{2}}
\end{aligned}
$$

Also straightforward computations lead to

$$
s_{x}=\frac{1}{\sqrt{\varphi}} \frac{\beta}{1+a u}\left(u_{x}-\sqrt{\varphi} v_{x}\right)=\frac{\left(u_{x}-\sqrt{\varphi} v_{x}\right)}{1+a u-a v \sqrt{\varphi}}
$$


By combining (3.17), (3.18), and (3.19), we arrive at

$$
\partial_{t} W=\varphi^{-5 / 4} \frac{\varphi^{\prime}}{4}\left(1-a^{2}\left(\frac{v}{1+a u}\right)^{2} \varphi\right) W^{2} .
$$

If we choose $\delta$ sufficiently small, the coefficient of the quadratic term in (3.20) remains bounded away from zero; that is, $\varphi^{-5 / 4} \varphi^{\prime}\left(1-a^{2}(v /(1+a u))^{2} \varphi\right) / 4 \geq k>0$. Consequently, (3.20) gives

$$
\partial_{t} W \geq k W^{2}
$$

Therefore, by choosing initial data small enough and satisfying (3.4) with derivatives such that $W_{0}>0,(3.21)$ shows that $W$ (hence $r_{x}$ ) blows up in finite time. This completes the proof.

REMARK 3.3. Similar result can be obtained for $\varphi^{\prime}(0)<0$. In this case consider the evolution of $s_{x}$ on the forward characteristics.

REMARK 3.4. A simple integration of (3.21) shows that the larger $W_{0}$ is, the quicker the blowup takes place.

ACKNOWLEDGEMENTS. The author would like to express his sincere thanks to King Fahd University of Petroleum and Minerals (KFUPM) for its support. Also special thanks go the referee for his valuable suggestions.

\section{REFERENCES}

[1] C. M. Dafermos and W. J. Hrusa, Energy methods for quasilinear hyperbolic initialboundary value problems. Applications to elastodynamics, Arch. Rational Mech. Anal. 87 (1985), no. 3, 267-292. MR 86k:35086. Zbl 586.35065.

[2] T. Kato, The Cauchy problem for quasi-linear symmetric hyperbolic systems, Arch. Rational Mech. Anal. 58 (1975), no. 3, 181-205. MR 52\#11341. Zbl 343.35056.

[3] W. Kosiński, Gradient catastrophe in the solution of nonconservative hyperbolic systems, J. Math. Anal. Appl. 61 (1977), no. 3, 672-688. MR 57\#903. Zbl 369.35043.

[4] P. D. Lax, Development of singularities of solutions of nonlinear hyperbolic partial differential equations, J. Mathematical Phys. 5 (1964), 611-613. MR $29 \# 2532$. Zbl 135.15101.

[5] T.-T. Li, Y. Zhou, and D.-X. Kong, Weak linear degeneracy and global classical solutions for general quasilinear hyperbolic systems, Comm. Partial Differential Equations 19 (1994), no. 7-8, 1263-1317. MR 95g:35111. Zbl 0810.35054.

[6] _ Global classical solutions for general quasilinear hyperbolic systems with decay initial data, Nonlinear Anal. 28 (1997), no. 8, 1299-1332. MR 98a:35082. Zbl 874.35068.

[7] R. C. MacCamy and V. J. Mizel, Existence and nonexistence in the large of solutions of quasilinear wave equations, Arch. Rational Mech. Anal. 25 (1967), 299-320. MR 35\#7000. Zbl 146.33801.

[8] S. A. Messaoudi, Formation of singularities in heat propagation guided by second sound, J. Differential Equations 130 (1996), no. 1, 92-99. MR 97f:35137. Zbl 0864.35015.

[9] _ Formation of singularities in solutions of a wave equation, Appl. Math. Lett. 12 (1999), no. 4, 23-28. MR 2000k:35202. Zbl 942.35116.

[10] S. Nishibata, The initial-boundary value problems for hyperbolic conservation laws with relaxation, J. Differential Equations 130 (1996), no. 1, 100-126. MR 97i:35113. Zbl 0872.35065 . 
[11] M. Slemrod, Instability of steady shearing flows in a nonlinear viscoelastic fluid, Arch. Rational Mech. Anal. 68 (1978), no. 3, 211-225. MR 80c:76004. Zbl 393.76004.

[12] Y.-S. Zheng, Vacuum problem for the damped p-system, Nonlinear Evolutionary Partial Differential Equations (Beijing, 1993), AMS/IP Stud. Adv. Math., vol. 3, American Mathmatical Society, Rhode Island, 1997, pp. 633-637. MR 98f:35094. Zbl 891.35087.

S. A. Messaoudi: Mathematical Sciences Department, King Fahd University of Petroleum AND Minerals, DHAHRAN 31261, SAUdi ARABIA

E-mail address: messaoud@kfupm. edu . sa 


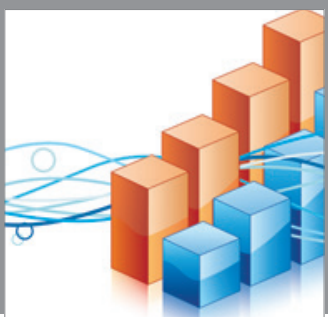

Advances in

Operations Research

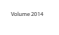

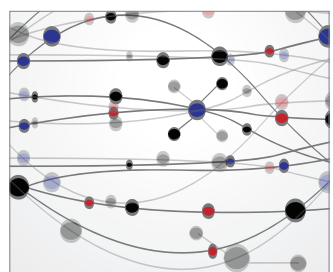

\section{The Scientific} World Journal
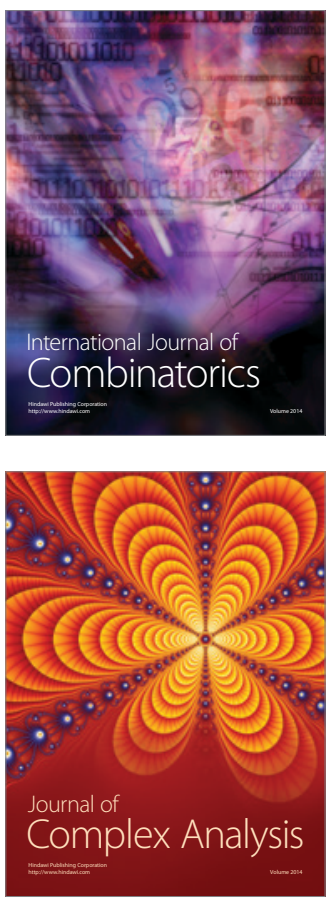

International Journal of

Mathematics and

Mathematical

Sciences
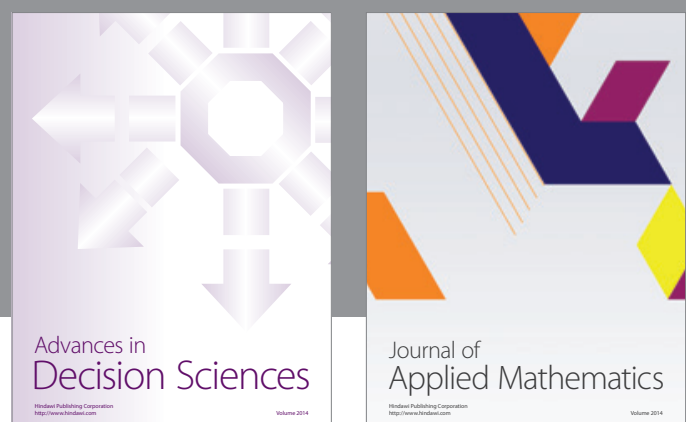

Journal of

Applied Mathematics
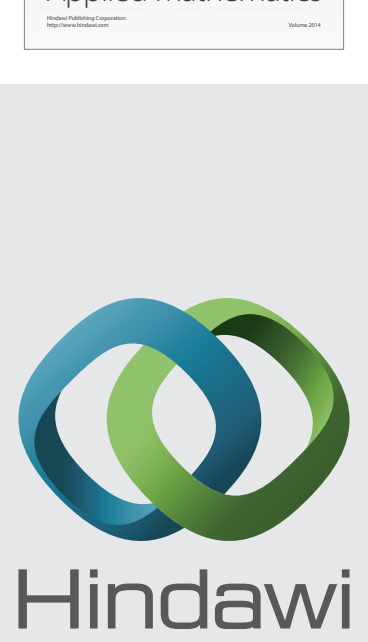

Submit your manuscripts at http://www.hindawi.com
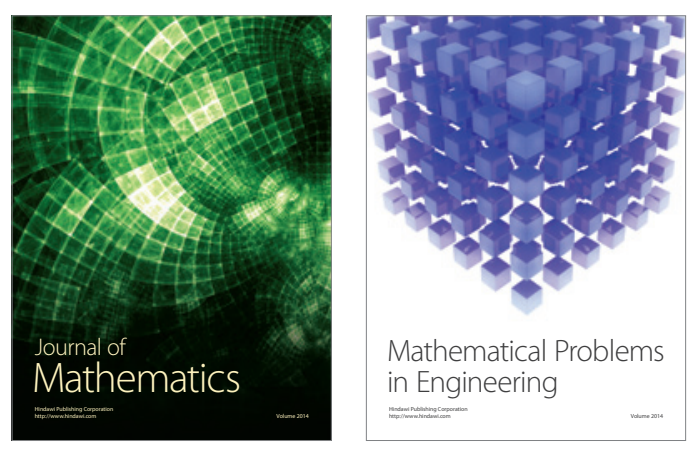

Mathematical Problems in Engineering
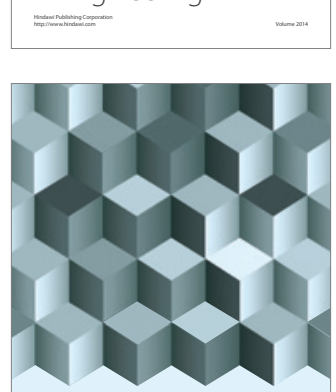

Journal of

Function Spaces
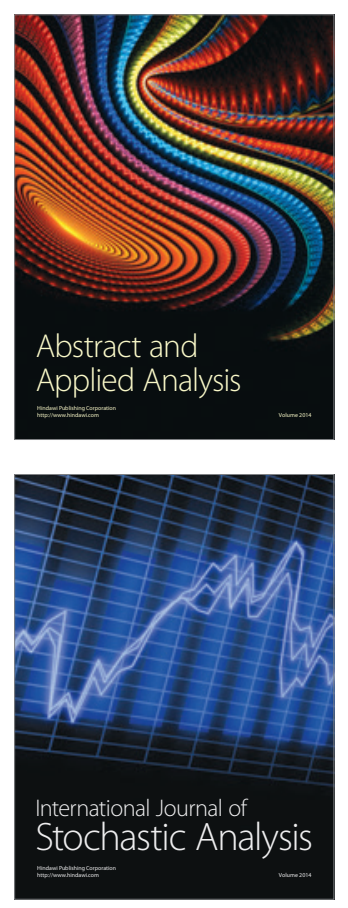

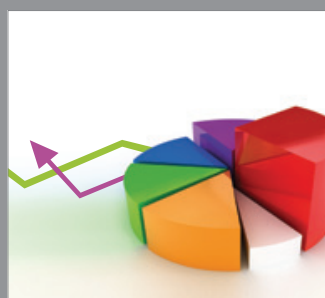

ournal of

Probability and Statistics

Promensencen
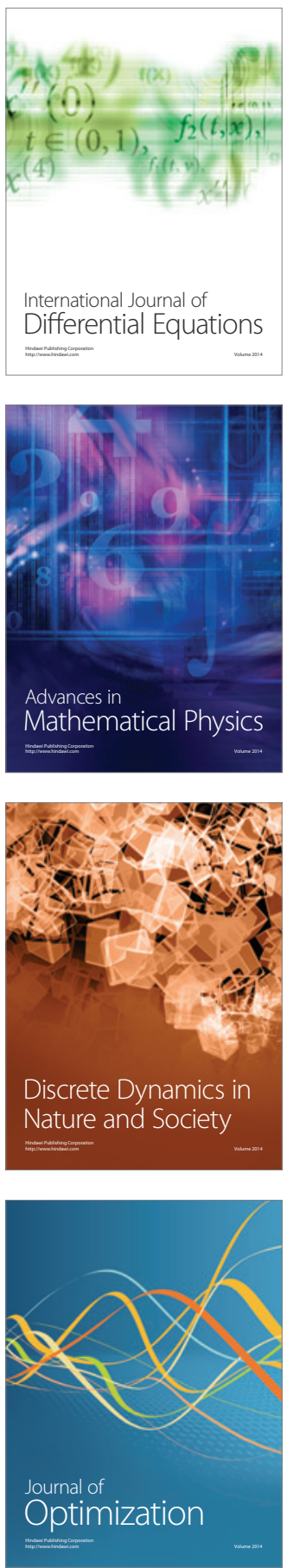